\title{
Multi-frequency HF radar measurements of artificial F-region field-aligned irregularities
}

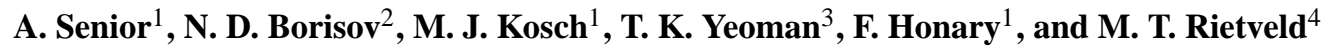 \\ ${ }^{1}$ Dept. of Communication Systems, Lancaster University, Lancaster, LA1 4WA, UK \\ ${ }^{2}$ IZMIRAN, 142190 Troitsk, Moscow Region, Russia \\ ${ }^{3}$ Dept. of Physics and Astronomy, University of Leicester, University Road, Leicester, LE1 7RH, UK \\ ${ }^{4}$ EISCAT Scientific Association, Ramfjordmoen, 9027 Ramfjordbotn, Norway
}

Received: 25 March 2003 - Revised: 5 July 2004 - Accepted: 15 July 2004 - Published: 3 November 2004

\begin{abstract}
We present radar backscatter power measurements using the CUTLASS HF radar at Hankasalmi, Finland from F-region field-aligned irregularities induced by HF radio pumping with the EISCAT Heating facility. A novel radar operating mode is used in which the radar frequency is rapidly swept through a number of bands, making use of the varying ionospheric refraction to probe different heights within the heated region. We obtain height profiles of backscatter power which correspond to $e$-folding scale lengths of around $20 \mathrm{~km}$ for the mean-square electron density perturbations for pump wave interaction heights in the region of $240-250 \mathrm{~km}$ in daytime conditions. The results are in agreement with previous measurements made by other techniques. We discuss some problems with the method and suggest improvements for future experiments.
\end{abstract}

Key words. Ionosphere (active experiments; ionospheric irregularities; instruments and techniques)

\section{Introduction}

Artificial field-aligned irregularities (AFAI) are one of the most important effects produced by the interaction of a high power "heater" or "pump" high frequency (HF) wave with the F-region ionosphere (Gurevich, 1978; Robinson, 1989). In the vicinity of the layer where the pump frequency matches the local plasma upper-hybrid frequency, the pump wave can couple to upper-hybrid (UH) waves. These UH waves are trapped in plasma density irregularities (Mjølhus, 1983) and heat the plasma there, causing the irregularities to deepen. In the magnetised F-region plasma, field-parallel (longitudinal) transport greatly exceeds field-transverse transport and leads to the irregularities becoming greatly elongated along the magnetic field-lines. These highly elongated filaments of depleted plasma density and enhanced electron temperature with small-scale sizes transverse to the magnetic field are often referred to as

Correspondence to: A. Senior

(a.senior@lancaster.ac.uk) "striations". Factors such as the anomalous absorption of the pump wave (Robinson, 1989), the nonlinear increase of longitudinal thermal conductivity (Gurevich et al., 1995) and the influence of the plasma distribution within the irregularities on coupling of the pump wave to UH waves (Borisov and Robinson, 2003) are expected to be responsible for the stabilisation of the instability.

The fluctuations in electron density caused by the presence of these striations form an effective target for radar backscattering. Backscatter from AFAI was first observed at HF and VHF by Thome and Blood (1974), where the irregularities were generated using the Platteville ionospheric heater in Colorado, USA. These early observations established that the backscatter was highly aspect-sensitive, with backscatter being observed only when the radar beam was nearorthogonal to the magnetic field lines in the heated region. Fialer (1974) reported that the backscatter power fell by at least $20 \mathrm{~dB}$ when the deviation from orthogonality reached $3^{\circ}$ and Minkoff et al. (1974) found that attempting to measure the vertical extent of the irregularities by varying the elevation angle of the radar beam resulted in nothing more than a measurement of the radar's radiation pattern in elevation!

Further radar backscatter measurements established the field-transverse wave number spectrum of the irregularities (Minkoff, 1974). These observations have been confirmed by in-situ rocket measurements (Kelley et al., 1995; Franz et al., 1999). The spectrum is essentially flat with increasing wave number up to a first "break-point" at scales of a few metres to tens of metres when an inverse power-law dependence takes over. Another break-point occurs at higher wave numbers where the power-law index changes. Franz et al. (1999) showed that this spectral form for their in-situ measurements made at Arecibo was similar to the Platteville results of Minkoff (1974), except that the break-points occurred at a higher wave number at Platteville. Franz et al. (1999) speculated that the difference may be due to the differing magnetic dip angle at the two locations and that higher dip angles may lead to the break-points moving to higher wave numbers. 


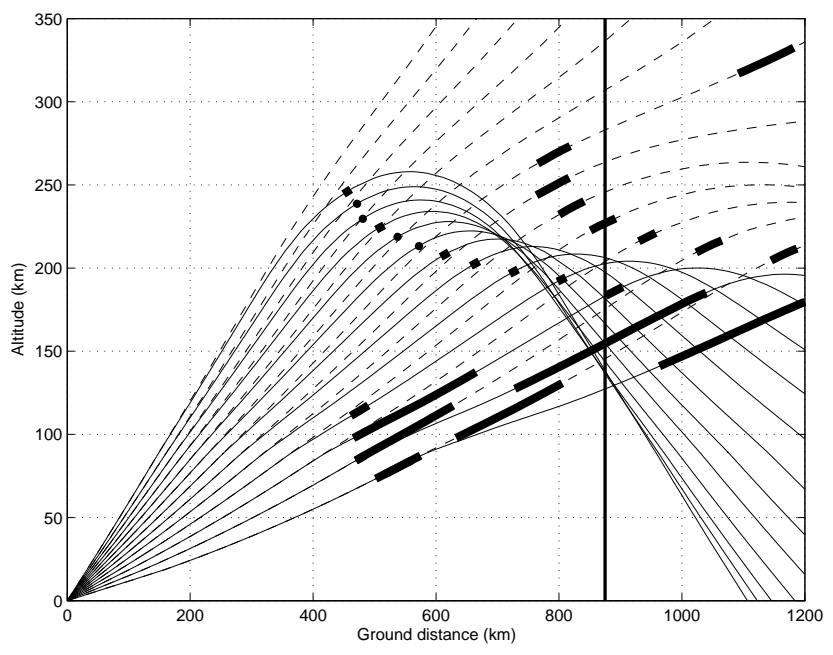

Fig. 1. The paths of HF rays transmitted from the Hankasalmi radar through the heated volume. Frequencies of 12.4 (solid lines) and 19.5 $\mathrm{MHz}$ (dashed lines) are shown for a range of elevation angles between $6^{\circ}$ and $30^{\circ}$ in steps of $2^{\circ}$. The regions where the rays lie within $1^{\circ}$ of orthogonality to the magnetic field line are marked by heavy lines. These regions are only marked for group ranges corresponding to the field-of-view of the CUTLASS radar used in the present experiment $(\sim 480-1590 \mathrm{~km})$. The vertical line marks the ground distance of the Troms $\varnothing$ heater from the radar.

Radar measurement of the vertical, or rather field-parallel, characteristics of the irregularities has proved difficult because of the extreme aspect sensitivity described above. Insitu measurements by rocket would require the rocket to follow a near-vertical trajectory in the heated region which is not easy to achieve due to constraints on launch and landing sites near heating facilities. Consequently, observations of the vertical extent of the irregularities are less numerous and less consistent. Fialer (1974) was able to estimate the vertical extent from radar measurements by allowing the heater interaction height (where irregularities are expected to be strongest) to vary, causing backscatter to be obtained from a range of heights relative to the interaction height and concluded that the Gaussian semi-thickness of the layer was about $7.5 \mathrm{~km}$ at Platteville. Korovin et al. (1983) determined that the thickness of the layer was about $10 \mathrm{~km}$ by performing spatial and frequency correlation measurements on radio waves scattered from the heated region. Jones et al. (1984) made measurements of the anomalous absorption of HF waves passing through the ionosphere heated by the Troms $\varnothing$ heater and concluded that the $e$-folding vertical scale of the mean-square electron density perturbation in the irregularities was on the order of $20 \mathrm{~km}$. Robinson (1989) gives a figure of $52 \mathrm{~km}$ for another anomalous absorption experiment at Troms $\varnothing$.

Hedberg et al. (1983) used a backscatter radar at Kiruna, Sweden, to observe irregularities generated by the Troms $\varnothing$ heater. They used frequencies of 3 and $7 \mathrm{MHz}$ and, by virtue of the difference in reflection heights at the two radar frequencies, estimated that the vertical extent of the irregularities, mainly below the interaction height, was around $80 \mathrm{~km}$ under daytime conditions. Using a similar technique with frequencies in the $2.7-4.8 \mathrm{MHz}$ range, Bakhmet'yeva et al. (1989) found the irregularities generated by the Sura heater to extend at least $50 \mathrm{~km}$ below the interaction height.

Measurements of the elongation of striations are important in assessing the accuracy of theories of their stationary state (Gurevich et al., 1995; Borisov et al., to appear in J. Plasma Phys., $2004^{1}$ ). Such theories do not take into account the possible effects on plasma transport due to the excitation of plasma turbulence in the region where the pump wave interacts with the plasma. In addition, the small-scale irregularities involve steep gradients in plasma density which may be unstable to, for example, the drift-wave instability, as has been suggested by Kelley et al. (1995). Such instabilities could prevent striations reaching the stationary state, as predicted by current theories. Both these factors could result in the elongation of striations being much less than these theories predict.

The experiment described here makes use of the capabilities of a modern coherent scatter radar to perform multifrequency HF backscatter measurements on the irregularities in an attempt to determine their variation with altitude, in a manner similar to Hedberg et al. (1983). By varying the HF radar frequency, it is possible to explore a range of heights throughout the heated volume and thereby to detect the existence and intensity of striations in the volume as a function of height. The principle is illustrated in Fig. 1 which shows the paths of rays at two different frequencies for a range of elevation angles. The regions where the ray falls within $1^{\circ}$ of orthogonality to the magnetic field direction are indicated.

\section{Instrumentation}

The European Incoherent Scatter (EISCAT) HF Heating facility (Rietveld et al., 1993) located at Ramfjordmoen near Troms $\varnothing$, Norway $\left(69.6^{\circ} \mathrm{N}, 19.2^{\circ} \mathrm{E}\right)$ was used to generate AFAI. An O-mode pump wave with an effective isotropic radiated power of $240 \mathrm{MW}$ was transmitted at an angle of $9^{\circ}$ south of the vertical in the magnetic meridian plane. The beam width (full width at half-maximum power) was $14.5^{\circ}$. The HF pump wave cycle was $30 \mathrm{~s}$ on $2.5 \mathrm{~min}$ off, with the on-time synchronised to a 3-min boundary of UT. Pump frequencies of 6.2 and $7.1 \mathrm{MHz}$ were used.

The EISCAT UHF radar (Rishbeth and van Eyken, 1993), co-located with the heater, was operated with the beam fieldaligned in the F-region (12.9 ${ }^{\circ}$ south of vertical in the magnetic meridian plane) to make measurements of the ionospheric plasma parameters, most importantly the electron density profile.

Whilst the radar is capable of accurately measuring the shape of the electron-density profile, including "valleys"

\footnotetext{
${ }^{1}$ Borisov, N., Senior, A. and Honary, F.: Stationary state and relaxation of artificial irregularities excited in ionospheric heating experiments, to appear in J. Plasma Phys., 2004.
} 
which may be unseen by an ionosonde, the measurement is not absolute and must be calibrated, for example, by comparing the peak plasma frequency with that measured by an ionosonde $\left(f_{o} \mathrm{~F}_{2}\right)$. Accurate absolute electron densities are important for the ray-tracing analysis used to assess the region of the ionosphere viewed by the CUTLASS coherent scatter radar. The EISCAT Dynasonde and the Troms $\varnothing$ Digisonde (both co-located with the heater) were used to provide this calibration. The Dynasonde made soundings at 6min intervals and the Digisonde at 15-min intervals.

In order to detect the electron density perturbations of the striations, the CUTLASS (Co-operative UK Twin Located Auroral Sounding System) HF coherent scatter radar (Milan et al., 1997b) at Hankasalmi, Finland $\left(62.3^{\circ} \mathrm{N}, 26.6^{\circ} \mathrm{E}\right)$ was employed. The radar can operate two different modes simultaneously (Lester et al., 2004), the two modes being referred to as channels A and B. In this paper we analyse data from channel B only which operated a single beam (beam 5) that passes approximately through the centre of the heated region. The radar frequency on channel B was stepped through a set of frequency bands of widths $\leq 265 \mathrm{kHz}$ covering part of the radar's frequency range $(8-20 \mathrm{MHz})$, the band being changed every second. The exact frequency used within a band is chosen automatically by the radar on the basis of least interference. Both channels measured 75 range gates at $15-\mathrm{km}$ spacing, with the nominal range to the first gate at $480 \mathrm{~km}$. Allowing for the receiver propagation delay of $100 \mu \mathrm{s}$, the range to the centre of the first gate is in fact $457.5 \mathrm{~km}$. These corrected ranges were used in the data analysis. Figure 2 depicts the experimental configuration used.

For each beam sounded, the CUTLASS radar records the parameters resulting from functional fits to the autocorrelation function of the received signal in each range gate, as well as various system parameters. In this experiment we are interested in measuring the backscatter power, since this is proportional to the mean-square electron density variation in the irregularities. The radar does not record backscatter power but signal-to-noise ratio (SNR). To obtain backscatter power we must multiply the SNR by the noise level and make an allowance for the receiver attenuator setting. Here we stop short of making an absolute calibration of the power and quote powers in decibels relative to an arbitrary reference.

The radar antenna gain increases with frequency. For an elevation angle of $10^{\circ}\left(15^{\circ}\right)$ the gain increases by about $5 \mathrm{~dB}$ $(3 \mathrm{~dB})$ from $12-20 \mathrm{MHz}$. Since the same antenna is used for transmission and reception, this amounts to an increase of $10 \mathrm{~dB}(6 \mathrm{~dB})$ from $12-20 \mathrm{MHz}$. This is significant compared to the observed variation in power with frequency, and we compensate for it by subtracting twice the antenna gain in $\mathrm{dB}$ relative to an isotropic radiator from the measurements, giving a "corrected power" which is effectively what would be measured if the antenna was an isotropic radiator. The correction applied is obtained from the antenna radiation pattern based on both the operating frequency and the elevation angle of arrival of the backscatter signal estimated from ray tracing. In addition, since we are considering volume

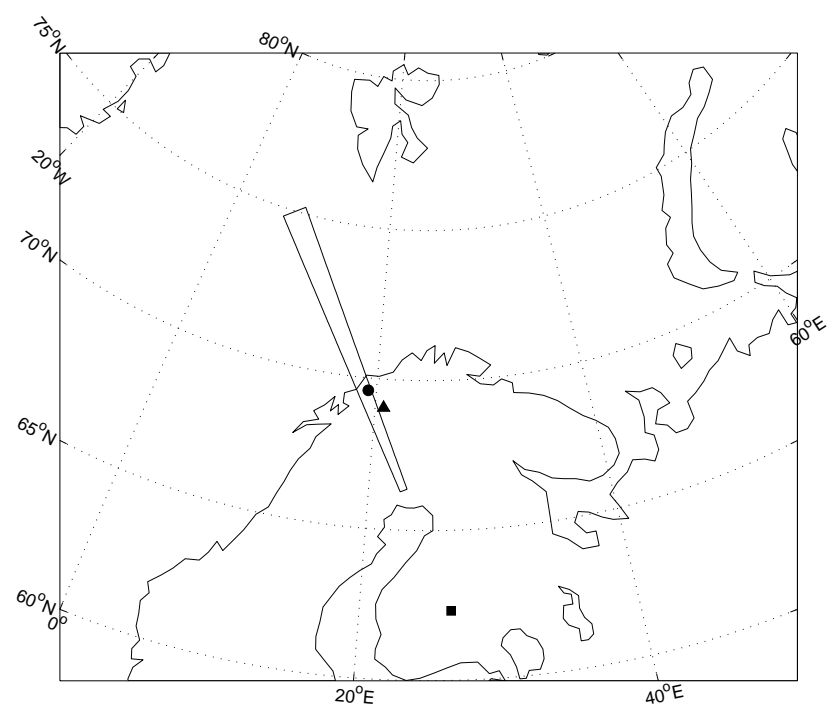

Fig. 2. The locations of the instruments used. The square indicates the CUTLASS Hankasalmi radar and the circle the EISCAT Troms $\varnothing$ site. The triangle marks the imaging riometer at Kilpisjärvi. Also shown is the field-of-view of beam 5 of the Hankasalmi radar used in the experiment.

scattering, we must also take into account the variations in antenna beamwidth, since these determine how the size of our scattering volume varies. The aspect sensitivity of the irregularities means that, to a good approximation, the target does not fill the beam in elevation and therefore we may neglect the variation of the beamwidth in elevation (from about $25^{\circ}$ at $12 \mathrm{MHz}$ to $14^{\circ}$ at $20 \mathrm{MHz}$, at $-3 \mathrm{~dB}$ relative to the peak). We cannot neglect the variation in azimuth since the target does indeed fill the beam in this plane; even though the heater beamwidth itself (at $-3 \mathrm{~dB}$ ) when projected to F-region heights may not fill the radar beam in azimuth, the irregularities are excited over a much larger area and are typically observed in at least the two beams adjacent to beam 5 (Bond et al., 1997). The azimuthal beamwidth (at $-3 \mathrm{~dB}$ ) decreases from $5.6^{\circ}$ at $12 \mathrm{MHz}$ to $3.2^{\circ}$ at $20 \mathrm{MHz}$ and is in fact almost exactly inversely proportional to frequency. We correct our observed powers for azimuthal beamwidth by subtracting the logarithmic beamwidth (in $\mathrm{dB}$ ) from the powers. No correction for range has been applied to the data: the heater-induced backscatter was only received over the range interval approximately $900-1000 \mathrm{~km}$ (see Fig. 3). The backscatter power would be expected to fall as the inverse square of range in the case of a fully beam-filling target or as the inverse fourth-power of range in the case of a point target. Our case will fall somewhere between these limits, but the difference in range-correction between 900 and $1000 \mathrm{~km}$ would amount to only $1.8 \mathrm{~dB}$ even in the case of an inverse fourth-power dependence on range.

For the purpose of these corrections, the antenna radiation patterns were computed using the commercial EZNEC software package which is based on the NEC-2 (Numerical Electromagnetics Code) computation engine. The ground 


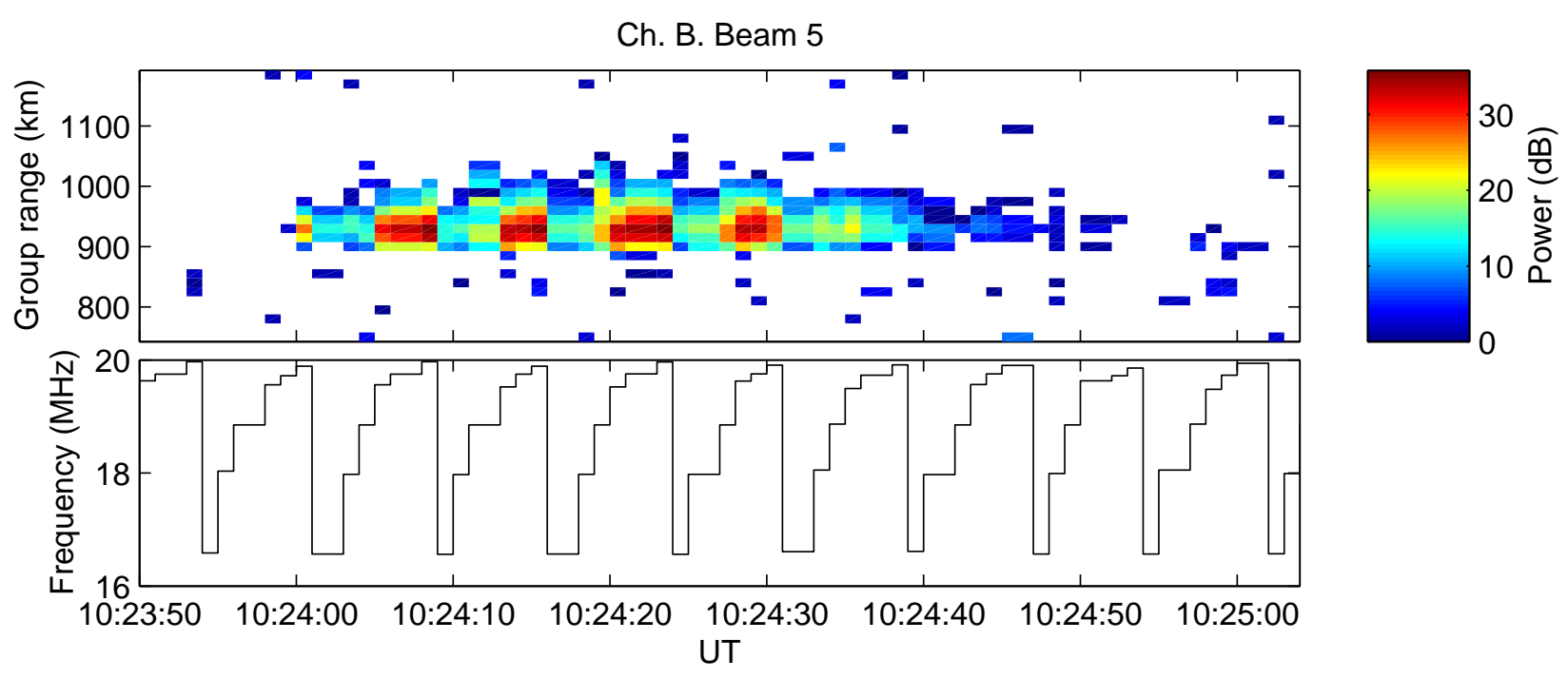

Fig. 3. Sample of radar backscatter power observations during a HF pump cycle. The top panel shows the backscatter power (dB) and the bottom panel the radar frequency (MHz). The HF pump was turned on at 10:24:00 UT and off at 10:24:30 UT on 5 November 2002. The AFAI backscatter can be seen between ranges of 900 and $1000 \mathrm{~km}$.

was modelled as having a conductivity of $7.5 \times 10^{-3} \mathrm{~S} \mathrm{~m}^{-1}$ and a dielectric constant of 12 . The version of software used was limited in its ability to handle complicated antenna systems like the CUTLASS array and so the modelling was performed in two stages by first computing the radiation pattern of a single log-periodic antenna of the type used in the array and then by computing the radiation pattern of an array of resonant half-wavelength dipoles having the same height and spacing as the antennae in the CUTLASS array. The results were then combined to give the radiation pattern of the array of log-periodic antennae as used by CUTLASS.

The gain of the receiver is reasonably constant over the range $9-20 \mathrm{MHz}$, varying by $1 \mathrm{~dB}$ or less. The gain is down by about $4 \mathrm{~dB}$ at $8 \mathrm{MHz}$ due to a steep filter cutoff to prevent interference from HF broadcast stations. Since we do not use frequencies below $10 \mathrm{MHz}$, we make no correction for receiver gain. Similarly, the attenuation due to the antenna phasing network used to form the beam for reception increases by only $0.6 \mathrm{~dB}$ over the range $8-20 \mathrm{MHz}$ and we make no correction for this. We assume that the transmitter power remains constant across the frequency range.

\section{Data analysis and results}

Several runs of the experiment were carried out in the period 2-8 November 2002 . The best quality data were taken on 5 November 2002 and 8 November 2002. However, for reasons to be addressed later, in this paper we will analyse results from 11 November 2002 only, specifically the period 10:2411:00 UT.

A typical example of the radar observations during a pump cycle is shown in Fig. 3. Backscatter is observed in beam 5 between ranges of approximately 900 and $1000 \mathrm{~km}$ immediately after pump-on. The backscatter is observed on several frequencies but with different intensities, leading to the characteristic cyclic pattern in the colours on the plot. The backscatter fades gradually after pump-off. This growth and relaxation behaviour is typical of that observed in such experiments (Frolov et al., 1997). Since we are interested in the variation in backscatter power with frequency and since the same radar frequency band is visited several times during one pump-on period, we may reduce the data by averaging together the backscatter powers observed on the same frequency band during the same pump-on period. As noted earlier, the exact frequency used in a band may vary, but since the bands have widths of between 15 and $265 \mathrm{kHz}$, we treat all frequencies in the same band as being the same frequency, taken to be equal to the centre frequency of the band. Furthermore, since we are concerned with the stationary state of the irregularities, we neglect the first $10 \mathrm{~s}$ of each pump-on period to reduce contamination from the growth phase. The result of this frequency binning is shown in the left panel of Fig. 4 where it has been applied to the pump-on period in Fig. 3.

\subsection{Mapping of the radar data using ray tracing}

In order to interpret the observations, it is essential to know where the radar backscatter comes from in the ionosphere. To answer this question, a ray-tracing analysis was carried out. The ionospheric electron density profile obtained from the UHF radar for the time of the pump cycle under consideration was used for this purpose. This carries an implicit assumption that the profile remains correct over the entire ray path from the CUTLASS radar to the scattering volume. Since this is a distance of around $900 \mathrm{~km}$, this is unlikely to be correct but we may note that the rays in which we are interested do not actually enter the ionosphere until approximately half-way along the path, see Fig. 1. The ray tracing 


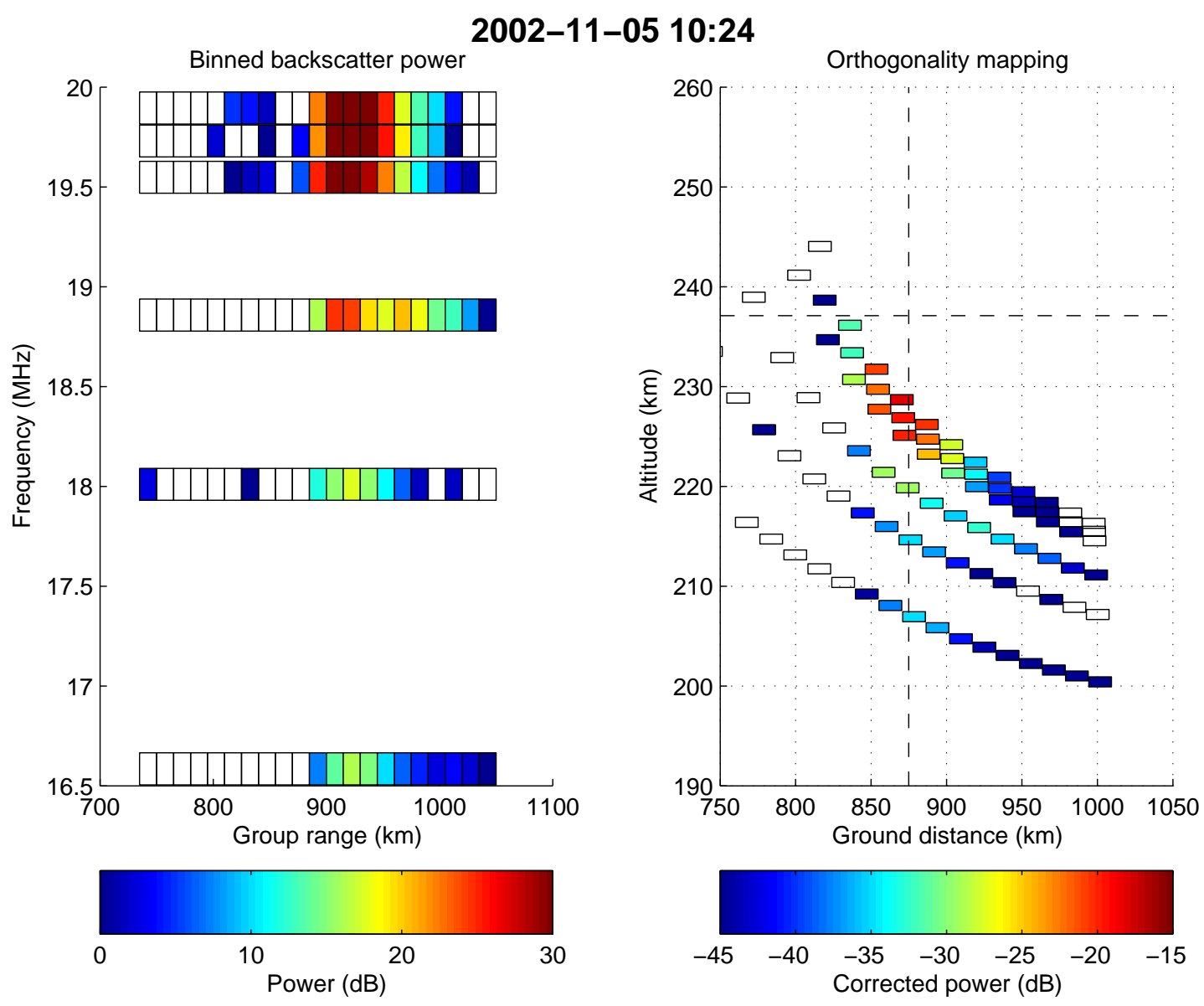

Fig. 4. Left panel: Backscatter power obtained by binning the backscatter power in Fig. 3 by frequency. Radar range gates where no scatter was detected are shown as empty boxes. Right panel: the data from the left panel mapped to the locations in the ionosphere where the scattering is occuring, assuming exact orthogonality. This mapping is carried out by means of ray-tracing results like those in Fig. 1. The horizontal dashed line indicates the upper-hybrid resonance height, determined from the electron density profile. The vertical dashed line indicates the ground distance of the heater from the CUTLASS radar. The power has been corrected for antenna gain and azimuthal beamwidth.

was carried out using a modified version of the Jones 3-D program (Jones and Stephenson, 1975). A dipole magnetic field model was used for speed in the ray tracing. To determine the angle of the radar wave from orthogonality at a given point, the wave propagation vectors were compared with the magnetic field direction obtained from the International Geomagnetic Reference Field (IGRF) model for 2002 (extrapolated from IGRF 2000) which provides a more accurate model than a dipole approximation. No significant difference was found by using the IGRF field in the ray tracing itself, as might be expected since the radar wave propagation is near-transverse to the magnetic field and is not greatly affected by it (Davies, 1990). Using the results of the ray tracing we can estimate the altitude and ground distance from the transmitter at which exact orthogonality is reached for a given radar frequency and group range, which is what the CUTLASS radar measures. This enables us to map the radar range gates to the locations in the ionosphere from which the backscatter is assumed to originate.
The right panel of Fig. 4 demonstrates this mapping for the data shown in the left panel of the figure. As expected, the higher frequencies map to higher altitudes in the ionosphere due to lower refraction. The loci of orthogonality slope downwards with increasing range as rays of a given frequency having lower take-off angles reflect at lower altitudes. We can see that the backscatter power increases with altitude towards the upper-hybrid resonance height. We can also note that the region of strongest backscatter is biased towards shorter distances from the radar. This may be because the pump beam was directed south of the zenith (slightly towards the CUTLASS radar). Similar effects were reported by Dhillon (2002). It is also possible that the backscatter is weaker at greater ranges because it originates at lower altitudes where the irregularities are weaker.

This mapping procedure works well in most cases, but occasionally problems are encountered. Figure 5 shows one such case. The two highest frequencies show strong scatter in gates which are not mapped in the right-hand panel of the 

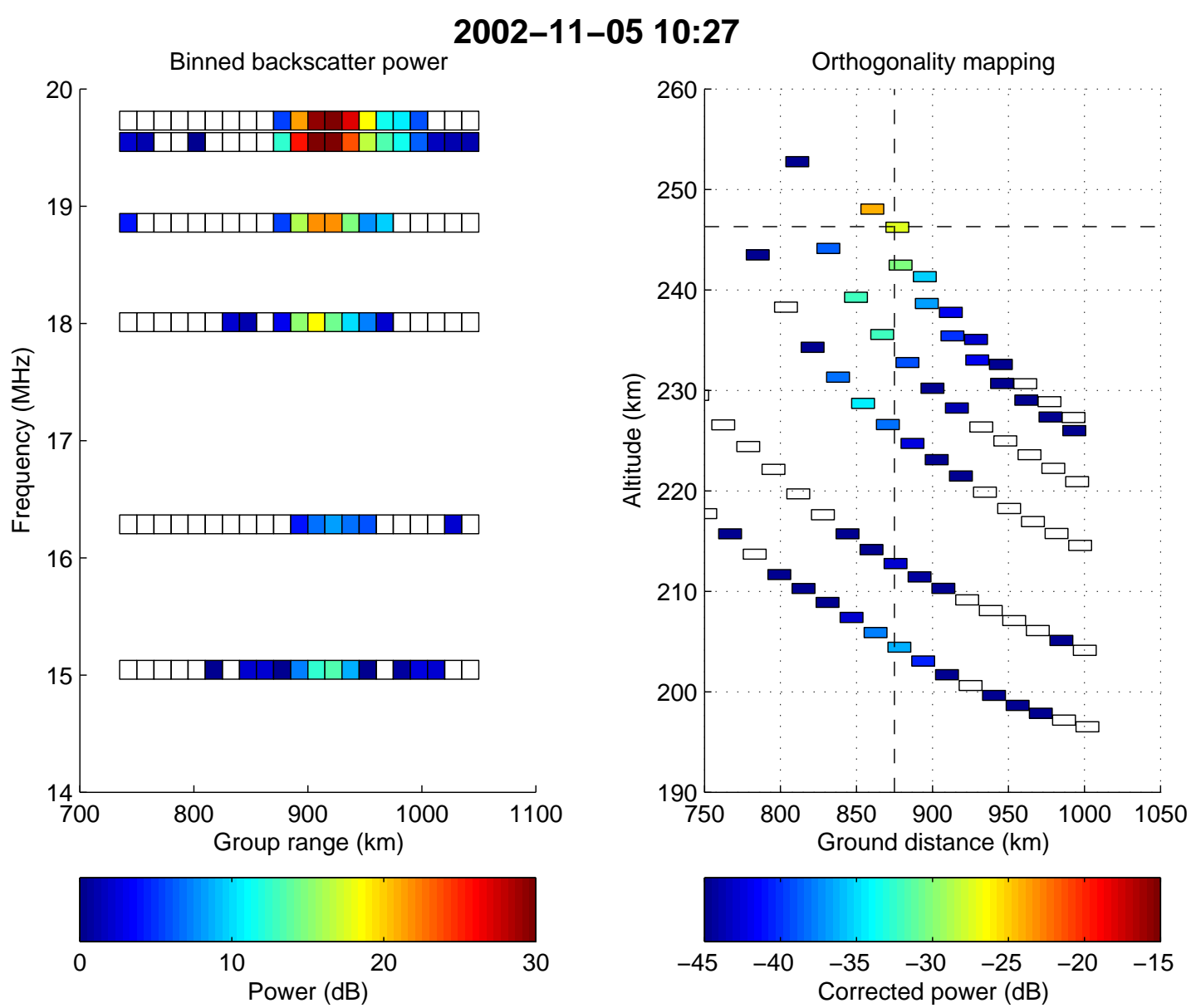

Fig. 5. The same as Fig. 4 but for the pump cycle beginning at 10:27 UT. Note that on the two highest frequencies, not all of the gates where strong scatter is detected in the left panel are mapped in the right panel.

figure because the ray-tracing analysis fails to find orthogonality at the group ranges of these gates on the relevant frequencies. There are at least two possible explanations for this discrepancy. Firstly, the scatter might be occuring at angles slightly off exact orthogonality, but considering the previous observations cited earlier, this seems unlikely. Secondly and more probably, the model of the ionospheric electron density used in the ray tracing, based on the single measurement at the end of the path near Tromsø, is not always a sufficiently accurate description of the ionosphere for the ray tracing to reproduce the observations exactly.

\subsection{Summary of results}

Having described the method of analysis applied to the observations from each pump cycle, we now summarise the results from the experimental run of 5 November 2002, 10:00-11:00 UT. Figure 6 shows a time series of height profiles of backscatter power obtained from successive pump cycles. The profiles are extracted from the orthogonalitymapped range gates by identifying the gate having the largest backscatter power over all frequencies and then choosing all gates having the same group range as this gate. The result- ing profiles are quasi-vertical through the heated region, as can be seen by considering Figs. 4 and 5. This procedure was adopted as a simple alternative to performing a twodimensional interpolation across the gates to give an exact vertical or field-aligned profile. Indicated on the plot are the the upper-hybrid resonance heights. It is clear from the plot that the increase in backscatter power with height is a consistent feature. During the cycles starting in the period 10:2410:45 UT, the pump frequency was $6.2 \mathrm{MHz}$, changing to 7.1 MHz from 10:48 UT. The increase in the reflection height on switching to the higher frequency is clearly seen.

In the pump cycles between 10:24 and 10:45 UT the maximum (corrected) backscatter power is typically about $-25 \mathrm{~dB}$ and is found at or within about $10 \mathrm{~km}$ of the upperhybrid resonance height. For the later cycles the maximum is much less, around $-40 \mathrm{~dB}$ but is found more than $10 \mathrm{~km}$ below the upper-hybrid resonance height. In these later cycles it would appear that the CUTLASS radar is only viewing the lower "tail" of the irregularities and not the region close to the upper-hybrid resonance height itself, with greater heights being inaccessible because of the upper frequency limit of the radar. However, another explanation of the reduced backscatter power is possible. The nearby imaging 


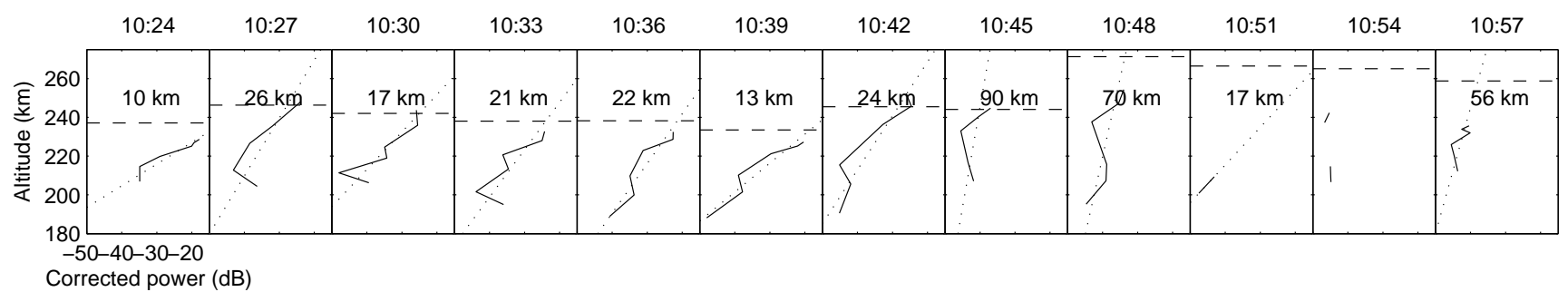

Fig. 6. Height profiles (solid lines) of backscatter power for all pump cycles during the period 5 November 2002 at 10:24-11:00 UT. The horizontal dashed lines indicate the upper-hybrid resonance heights. The diagonal dotted lines indicate linear fits to the profiles and the distances indicated in each panel are the $e$-folding lengths of the electron density perturbations derived from these fits.

riometer at Kilpisjärvi, Finland $\left(69.1^{\circ} \mathrm{N}, 20.8^{\circ} \mathrm{E}\right.$; see Fig. 2) observed an increased level of cosmic noise absorption at 38.2 $\mathrm{MHz}$ during the period 10:45-11:00 UT in the beam which intersects the D-region above the heater site. Although the absorption was only on the order of $0.5 \mathrm{~dB}$ at $38.2 \mathrm{MHz}$ this could mean substantial D-region absorption $(\sim 14 \mathrm{~dB})$ of the HF pump wave at $6-7 \mathrm{MHz}$ and a consequent reduction in its effect in the F-region. Before 10:45 UT the riometer absorption varied between 0.1 and $0.2 \mathrm{~dB}$.

Assuming that the backscatter power is proportional to the mean-square electron density perturbation in the irregularities, a linear fit to the logarithm of the power enables us to estimate the $e$-folding length of irregularities. These linear fits and scale lengths are shown in Fig. 6. The exponential decay model is only an approximation but appears reasonable for the profiles shown here. We see that in the period 10:2410:42 UT the scale lengths are similar, having a mean value of $19 \mathrm{~km}$ and a standard deviation of $6 \mathrm{~km}$. This is consistent with previous measurements at Troms $\varnothing$ during daytime conditions (Jones et al., 1984). After 10:45 UT the scale lengths apparently become much greater. As discussed earlier, this may be either because the CUTLASS radar is viewing only the tail of the irregularities or because the irregularities are simply weaker because of D-region absorption and the errors in the CUTLASS measurement are higher. In the 10:54 UT pump cycle, the linear fit had a negative slope (not shown), probably due to measurement uncertainties in the power.

\section{Discussion}

We have demonstrated how a coherent scatter HF radar may be used to examine the height profile of the electron density perturbations induced in the F-region by the action of a powerful electromagnetic wave. These observations were made in daytime conditions $(\sim 12: 00 \mathrm{LT})$ and are consistent with similar observations made by the anomalous absorption technique at Troms $\varnothing$ (Jones et al., 1984). The aim of the experiment was to study irregularities with small-scale sizes transverse to the magnetic field (striations). The radar technique is sensitive to fluctuations in the electron density with scales equal to half the radar wavelength. In the observations presented here, the radar frequency covered at most the range $13-20 \mathrm{MHz}$, corresponding to irregularity scales of 7.5-11.5 m, although as Kelley et al. (1995) pointed out, larger-scale irregularities with steep gradients in this range of lengths could also give rise to backscatter. The radar measurements of Hedberg et al. (1983) and Bakhmet'yeva et al. (1989) were made on relatively low frequencies corresponding to irregularity scales of $20-50 \mathrm{~m}$ and $30-50 \mathrm{~m}$, respectively. It is possible that at these larger scales, the radar is viewing irregularities generated by other mechanisms, for example, thermal self-focussing of the pump wave (Gurevich, 1978) rather than small-scale striations.

Since the lower frequencies reach orthogonality at lower altitudes, the radar views larger-scale irregularities at lower heights. If there is a significant dependence of field-parallel scale on field-transverse scale, this means that the observed profile of electron density perturbation is a combination of profiles for different transverse scale sizes. Jones et al. (1984) explained that the anomalous absorption technique is not as sensitive to the transverse scale spectrum of the irregularities and essentially measures the overall density perturbation. They suggested that simultaneous comparison of that method with the radar technique (as described here) might help identify what role the transverse scale spectrum plays, but so far such simultaneous experiments do not appear to have been carried out.

It is interesting to note that in all our observations, we only viewed the irregularities below the upper-hybrid resonance height. Referring to Fig. 6 it is clear that had we chosen lower pump frequencies and thus lower upper-hybrid resonance heights, we would have been able to view the irregularities both above and below the upper-hybrid resonance height. In the experiment described here, the pump frequency was increased with time as the ionospheric plasma frequency increased, resulting in the upper-hybrid resonance height remaining relatively close to the F-region plasma density peak. This situation makes it more difficult to achieve orthogonality in the region above the upper-hybrid resonance level. Considering Fig. 1, in order to reach orthogonality at these altitudes (above $250 \mathrm{~km}$, say), the rays must initially have relatively high elevation angles and therefore must undergo considerable refraction to reach orthogonality. Close to the F-region peak and into the topside, the refraction is either too weak or in the wrong direction to bring the rays to orthogonality. Using lower frequencies results in orthogonality 
being reached either at too low an altitude or too short a ground distance, whereas as the frequency is increased, rays at the higher elevation angles penetrate the ionosphere before reaching orthogonality.

In discussing Fig. 5 we pointed out an inconsistency between the coherent radar observations and the ray-tracing analysis, which is probably due to an inadequate model of the ionospheric electron density distribution along the path from the radar to the scattering volume. In attempting to analyse the data collected on 8 November 2002, during the period 15:00-17:00 UT, we found that this inconsistency was more severe, often resulting in the ray tracing indicating no orthogonality in gates on the higher radar frequencies which showed the strongest backscatter, suggesting that the raytracing model was underestimating the ionospheric refraction. This inevitably must also cast some doubt on the reliability of the ray tracing for the data presented here, even though no major discrepancies are observed in the majority of the cases. The accuracy of the ray tracing should be improved by means of more detailed information on the electron density distribution. This could be obtained by pointing the UHF incoherent scatter radar along the HankasalmiTroms $\varnothing$ path at different elevation angles and possibly supplemented by ionosondes in the vicinity of the path.

The CUTLASS radar is equipped with an interferometer to measure the elevation angle of arrival of the backscatter signal (Milan et al., 1997a). Unfortunately, technical problems with the radar meant that we were unable to interpret the elevation angle data in this experiment. These data would further allow us to test the accuracy of the ray tracing and even to confirm if the scatter we are observing truly is originating at different heights on different frequencies or if it originates from similar heights. In the latter case the variation in power with frequency may in fact be due to the varying aspect angle of the radar beam to the irregularities, contradicting our assumption of near-infinite aspect sensitivity, although as explained in the Introduction, this assumption seems reasonable. In future experiments, modifying the CUTLASS sounding mode to collect returns from meteor trail ionisation at near-ranges will allow for the calibration of the elevation angles: assuming that meteor ionisation occurs in a narrow height interval in the E-layer and that ionospheric refraction of the radar beam can be neglected (straight-line propagation), there follows a simple relationship between radar range to the meteor trails and elevation angle.

Some of the observations (e.g. Fig. 4) suggest that the frequency range scanned by the CUTLASS radar was sometimes too narrow to cover the whole height interval occupied by AFAI, since the backscatter was still quite strong at the lowest frequency. In future experiments, using a much wider range of frequencies, perhaps always the full $8-20 \mathrm{MHz}$ range of the radar, will ensure that we view the full extent of the irregularities. Furthermore, the use of several closely spaced frequency bands meant that we obtained measurements which are bunched in altitude rather than evenly distributed. This does not make optimal use of the radar but, on the other hand, there may be an advantage in confirming that the measurements are consistent between similar frequencies, indicating no anomalies in the radar system.

The method described here may be used to investigate differences in the scale-lengths of striations under different ionospheric conditions. This will be an important test of theories describing the plasma transport processes associated with striations, since the transport coefficients will depend on the electron density and the bulk electron heating which differs considerably between daytime and nighttime conditions: compare, for example, the electron temperature observations of Stocker et al. (1992) in daytime conditions with those of Rietveld et al. (2003) in nighttime conditions. In addition, our results have practical value in helping to understand the propagation factors involved in maximising the backscatter power observed from heater-induced irregularities. Heater-induced coherent radar backscatter has found important applications in the study of magnetospheric ultralow frequency waves using the CUTLASS and EISCAT facilities (Yeoman and Wright, 2001) and future work with this technique is planned using CUTLASS and the recently comissioned SPEAR heater-radar facility located on Svalbard inside the polar cap (Wright et al., 2000). It is clear from our analysis that one must choose the pump and radar frequencies carefully to ensure that the irregularities are viewed close to their greatest intensity whilst taking account of the radar antenna gain variations with frequency and elevation angle.

\section{Conclusions}

In summary, we have found that it is practical to use a HF coherent scatter radar to explore the height variation of the electron density perturbations in irregularities generated by radiowave pumping of the ionosphere. The results presented are in agreement with previous measurements by other techniques, giving $e$-folding scale lengths of $\sim 20 \mathrm{~km}$ for the density perturbations in the irregularities for upper-hybrid resonance heights of $240-250 \mathrm{~km}$ in daytime conditions. We have found that inaccuracies in our knowledge of the electron density distribution in the ionosphere affects our ability to map the location of the radar backscatter by means of ray tracing and suggest that this could be ameliorated by using the incoherent scatter radar to make measurements along the $\mathrm{HF}$ radar propagation path. Improvements in the choice of frequencies used on the HF radar and the availability of elevation angle measurements will further enhance the technique. By making similar observations in daytime and nighttime conditions, it should be possible to investigate the influence of the plasma transport coefficients on the field-parallel scale of the irregularities, which will be useful for testing theories of the stationary state of these irregularities.

Acknowledgements. We are grateful to P. Cannon of QinetiQ Ltd. for providing the Troms $\varnothing$ Digisonde data and to the staff of the World Data Centre at the Rutherford Appleton Laboratory (RAL), UK for manual scaling of the digisonde ionograms. Technical details of the CUTLASS radar were kindly provided by P. Chapman 
(Lancaster). We thank I. McCrea of the EISCAT Group, RAL for helpful discussions and support. A. S. is supported by a research studentship from the UK's Particle Physics and Astronomy Research Council (PPARC). CUTLASS is a PPARC UK National Facility operated by the University of Leicester. The Kilpisjärvi imaging riometer (IRIS) is funded by PPARC and operated by Lancaster University in conjunction with Sodankylä Geophysical Observatory. EISCAT is an International Association supported by Finland (SA), France (CNRS), the Federal Republic of Germany (MPG), Japan (NIPR), Norway (NFR), Sweden (VR) and the United Kingdom (PPARC).

The Editor in Chief thanks B. Bristow and another referee for their help in evaluating this paper.

\section{References}

Bakhmet'yeva, N. V., Goncharov, N. P., Ignat'yev, Y. A., Korotina, G. S., Tolmacheva, A. V., and Shavin, P. B.: The SpatialTemporal Characteristics of Inverse Scattering Signals from an Artificial Disturbance Region, Geomagn. Aeron., 29, 701-705, 1989.

Bond, G. E., Robinson, T. R., Eglitis, P., Wright, D. M., Stocker, A. J., Rietveld, M. T., and Jones, T. B.: Spatial observations by the CUTLASS coherent scatter radar of ionospheric modification by high power radio waves, Ann. Geophys., 15, 1412-1421, 1997.

Borisov, N. D. and Robinson, T. R.: Transformation of an EM pump wave into upper hybrid resonance oscillations in striations in a vertically inhomogenous ionosphere, Phys. Lett. A, 315, 126135, 2003.

Davies, K.: Ionospheric Radio, Peter Peregrinus Ltd., 1990.

Dhillon, R. S.: Radar studies of natural and artificial waves and instabilities in the auroral ionosphere, Ph.D. thesis, University of Leicester, UK, 2002.

Fialer, P. A.: Field-aligned scattering from a heated region of the ionosphere - Observations at HF and VHF, Radio Sci., 9, 923940, 1974.

Franz, T. L., Kelley, M. C., and Gurevich, A. V.: Radar backscattering from artificial field-aligned irregularities, Radio Sci., 34, 465-475, 1999.

Frolov, V. L., Erukhimov, L. M., Metelev, S. A., and Sergeev, E. N.: Temporal Behaviour of Artificial Small-scale Ionospheric Irregularities. Review of Experimental Results, J. Atmos. Solar-Terr. Phys., 59, 2317-2333, 1997.

Gurevich, A. V.: Nonlinear Phenomena in the Ionosphere, Springer Verlag, New York, 1978.

Gurevich, A. V., Lukyanov, A. V., and Zybin, K. B.: Stationary State of Isolated Striations Developed During Ionospheric Modification, Phys. Lett. A, 206, 247-259, 1995.

Hedberg, A., Derblom, H., Thidé, B., Kopka, H., and Stubbe, P.: Observations of HF Backscatter Associated with the Heating Experiment at Troms $\varnothing$, Radio Sci., 18, 840-850, 1983.

Jones, R. M. and Stephenson, J. J.: A versatile three-dimensional ray tracing computer program for radio waves in the ionosphere, Tech. Rep. OT 75-76, Office of Telecommunications, U.S. Department of Commerce, 1975.

Jones, T. B., Robinson, T. R., Stubbe, P., and Kopka, H.: Frequency Dependence of Anomalous Absorption Caused by High Power Radio Waves, J. Atmos. Terr. Phys., 46, 147-153, 1984.
Kelley, M. C., Arce, T. L., Salowey, J., Sulzer, M., Armstrong, W. T., Carter, M., and Duncan, L.: Density Depletions at the $10 \mathrm{~m}$ Scale Induced by the Arecibo Heater, J. Geophys. Res., 100, 17367-17376, 1995.

Korovin, A. V., Nasyrov, A. M., and Yagnov, N. N.: Spatial and Frequency Correlation of the Field Scattered by Small-scale Artificial Inhomogeneities of the Ionospheric F-layer, Radiophys. Quant. Electron., 26, 120-124, 1983.

Lester, M., Chapman, P. J., Cowley, S. W. H., Crooks, S. J., Davies, J. A., Hamadyk, P., McWilliams, K. A., Milan, S. E., Parsons, M. J., Payne, D. B., Thomas, E. C., Thornhill, J. D., Wade, N. M., Yeoman, T. K., and Barnes, R. J.: Stereo CUTLASS - A new capability for the SuperDARN HF radars, Ann. Geophys., 22, 459-473, 2004.

Milan, S. E., Jones, T. B., Robinson, T. R., Thomas, E. C., and Yeoman, T. K.: Interferometric evidence for the observation of ground backscatter originating behind the CUTLASS coherent HF radars, Ann. Geophys., 15, 29-39, 1997a.

Milan, S. E., Yeoman, T. K., Lester, M., Thomas, E. C., and Jones, T. B.: Initial backscatter occurrence statistics from the CUTLASS HF radars, Ann. Geophys., 15, 703-718, 1997 b.

Minkoff, J.: Radio frequency scattering from a heated ionospheric volume, 3, Cross section calculations, Radio Sci., 9, 997-1004, 1974.

Minkoff, J., Kugelman, P., and Weissman, I.: Radio Frequency Scattering from a Heated Ionospheric Volume, VHF/UHF Fieldaligned and Plasma-line Backscatter Measurements, Radio Sci., 9, 941-956, 1974.

Mjølhus, E.: On reflexion and trapping of upper-hybrid waves, J. Plasma Phys., 29, 195-215, 1983.

Rietveld, M. T., Kohl, H., Kopka, H., and Stubbe, P.: Introduction to ionospheric heating experiments at Troms $\varnothing-$ I, Experimental overview, J. Atmos. Terr. Phys., 55, 577-599, 1993.

Rietveld, M. T., Kosch, M. J., Blagoveshchenskaya, N. F., Kornienko, V. A., Leyser, T. B., and Yeoman, T. K.: Ionospheric electron heating, optical emissions, and striations induced by powerful HF radio waves at high latitudes: Aspect angle dependence, J. Geophys. Res., 108, 1141, doi:10.1029/2002JA009543, 2003.

Rishbeth, H. and van Eyken, A. P.: EISCAT - Early history and the first ten years of operation, J. Atmos. Terr. Phys., 55, 525-542, 1993.

Robinson, T. R.: The heating of the high latitude ionosphere by high power radio waves, Phys. Rep., 179, 79-209, 1989.

Stocker, A. J., Honary, F., Robinson, T. R., Jones, T. B., Stubbe, P., and Kopka, H.: EISCAT observations of large scale electron temperature and electron density perturbations caused by high power HF radio waves., J. Atmos. Terr. Phys., 54, 1555-1572, 1992.

Thome, G. D. and Blood, D. W.: First observations of RF backscatter from field-aligned irregularities produced by ionospheric heating, Radio Sci., 9, 917-921, 1974.

Wright, D. M., Davies, J. A., Robinson, T. R., Chapman, P. J., Yeoman, T. K., Thomas, E. C., Lester, M., Cowley, S. W. H., Stocker, A. J., Horne, R. B., and Honary, F.: Space Plasma Exploration by Active Radar (SPEAR): an overview of a future radar facility, Ann. Geophys., 18, 1248-1255, 2000.

Yeoman, T. K. and Wright, D. M.: ULF waves with drift resonance and drift-bounce resonance energy sources as observed in artificially-induced HF radar backscatter, Ann. Geophys., 19, 159-170, 2001. 